\title{
Grenzen und Freiheit - eine interdisziplinäre Entdeckung
}

\author{
Anne-Coralie Bonnaire \\ Technische Universität Chemnitz \\ Institut für Pädagogik
}

\begin{abstract}
„Aus Traditionen Grenzen überschreiten“ - so lautet das Leitmotiv unserer Alma Mater, der Universität Leipzig. Es steht für Offenheit, Interdisziplinarität, internationale Zusammenarbeit und Wissensaustausch. In Anlehnung an dieses Leitmotiv und angesichts der aktuellen politischen, gesellschaftlichen und wissenschaftlichen Lage wollten wir als Nachwuchswissenschaftler*innen interdisziplinär und fakultätsübergreifend dem Verständnis von „Grenzen \& Freiheit“ auf den Grund gehen. So lautet die These vom französischen Philosophen Régis Debray: mehr Freiheit bedeutet eigentlich mehr Grenzen, weil diese nötig sind, um sich selbst zu verstehen und zu definieren, bevor man in Kontakt mit anderen treten kann. In der vorliegenden Ausgabe vom arbeitstitel | Forum für Leipziger Promovierende verstehen sich die Grenzen als räumliche, disziplinäre und wissenschaftliche Trennungen. Ungebundenheit, Bewegungs- und Niederlassungsfreiheit scheinen zentrale Stichworte des 21. Jahrhunderts zu sein, so etwa die Niederlassungsfreiheit innerhalb der EU (Richtlinie 2004/38/EG), Freier Zugang zu Daten, Medienkonvergenz, Big Data oder soziale Medien bieten unbegrenzte Möglichkeiten, alles zu erfahren, was in der Welt die Menschheit bewegt.
\end{abstract}

Doch bedeutet das Überschreiten solcher Grenzen, wie beispielsweise unbegrenzter Zugang zu Meinungen und Wissenschaftsergebnissen, tatsächlich mehr Freiheit? Wird nicht eher, im Gegenteil, durch Überwachung im Namen der Sicherheit, mehr Kontrolle über den Menschen geschaffen? Hat nicht das Auflösen von Grenzen - zwischen Wissenschaft und \#Fake Science, Journalismus und \#Fake-News, Privatem und Öffentlichen, Realem und Digitalem - vielmehr zum Ergebnis, dass andere Freiheiten schwinden? Grenzen und Freiheit wohnen jeder Wissenschaft inne; Grenzen können nicht nur gesellschaftliche, geographische und politische Räume begrenzen, sondern auch mathematische (Grenzwerte, Schwellenwerte), psychologische (Intimsphäre) oder medizinische (Haut). Auch der Begriff Freiheit hat viele Gesichter, von der Idee der Autonomie eines Subjekts bis zu den Freiheitsgraden in der Statistik.

Im Wintersemester 2018/19 hatten wir für die Leipziger Promotionsvorträge Promovierende aufgerufen, sich mit der Thematik „Freiheit und Grenzen“ in ihrer Forschung auseinanderzusetzen und durften am 24. Januar 2019 spannende Vorträge zu deren Forschung hören. In

Erschienen in: arbeitstitel - Forum für Leipziger Promovierende, Band 7, Heft 1 (2019), S. 1. Lizenzierung CC-BY-SA 4.0. dieser 21. Auflage des arbeitstitel werden nun Beiträge veröffentlicht, die sich dieser interdisziplinären Frage widmen. Jan Quenstedt (Theologie) untersucht die Botschaft von neutestamentlichen Schriften für das menschliche Zusammenleben auf das Vorhandensein von Freiheit und ihren Grenzen. Julia Franke (Philosophie) behandelt die Möglichkeiten des Ausdruck- und Unausdrückbaren in Anlehnung an die These der ,Unsagbarkeit' des Inhalts von Moritz Schlick. Benjamin Reimann (Philosophie) setzt sich mit der Frage der Überwindung (inter-)disziplinärer Grenzen durch den Dialog von Tomasello und Aristoteles zur Frage der Vernunft des Menschen als sozialer Wesen und/oder umgekehrt, auseinander. Robert Reimer (Philosophie) untersucht den freien Willen anhand der Experimente von Benjamin Libet. Zu guter Letzt setzt sich Frau Ji Lie (Philologie) mit der Frage der Freiheit in der Wahl der Partner aus interkultureller Sicht auseinander. Sie nutzt hierfür einen Vergleich zwischen westlichen (am Beispiel Deutschland) und fernöstlichen (am Beispiel China) Werten, die an eine Ehe geknüpft sind. Die Autor*innen dieser $a r$ beitstitel-Ausgabe geben Anlass zur Perspektivwechsel und Einblicke in die aktuellste Forschung der Leipziger Nachwuchswissenschaftler*innen. Vielen Dank auch an Franziska Naether für ihr Grußwort.

Eine lange Tradition haben die Leipziger Promotionsvorträge und deren Veröffentlichungen in Form von $a r$ beitstitel; wir freuen uns sehr, diese, nach drei Jahren Pause, wieder ins Leben rufen zu dürfen. Wir bedanken allen bisherigen Mitwirkenden des Prorats und des arbeitstitel für die Übertragung der Verantwortlichkeit der neusten Editionen und die Gutachter*innen für den wissenschaftlichen Feedback zu den Einreichungen: KyeongHwa Lee (UL), Moritz Rudolph (UL), Dr. Franziska Naether (UL), Christine Stadler (TU Chemnitz), Maria Stuckenberg (UL). Auch für uns als neue Herausgeberschaft bietet die Mitarbeit an den Leipziger Promotionsvorträgen und der Zeitschrift arbeitstitel eine Chance, einen Blick über unseren bisherigen (wissenschaftlichen) Tellerrand werfen zu können und wir freuen uns auf die nächsten Promotionsvorträgen.

\section{Literatur}

Debray, R. (2010) Eloge des frontières, coll. Blanche, Gallimard, Paris.

Europäisches Parlament/Rat der Europäischen Union (2004) Richtlinie 2004/38/EG, Brüssel. 